\title{
Editorial
}

\section{Dietary Fibre and Diabetes}

\author{
T. J. Goulder and K. G. M. M. Alberti \\ Faculty of Medicine, Chemical Pathology and Human Metabolism, Level D, South Laboratory and Pathology Block, \\ General Hospital, Southampton, England
}

In recent years dietary fibre has received a great deal of attention in both the medical $[1,2,3]$ and the lay press. "Bran" has become the watchword of the "back-to-nature" brigade; and health food stores, newspapers and popular magazines alike encourage us to increase the roughage in our diets. The reasons for this enthusiasm can be found not only in the evangelical exhortations of the enthusiasts but also in the epidemiological and experimental observations which have attributed several major disease states to the low fibre content of diets in Western societies [4, $5,6,7]$. These disorders include coronary heart disease, diverticular disease and carcinoma of the colon, appendicitis, gall bladder disease, dental caries and, last but not least, diabetes mellitus. It is immediately apparent that it is difficult, if not impossible, to construct a unitary aetiological hypothesis linking these diverse diseases. It should be noted that diets high in fibre tend to be low in protein, fats and refined carbohydrates: whether it is the high fibre content per se, that is important, or associated changes in other constituents remains unclear. In all probability both components play a role.

Before discussing the potential impact of high fibre diets on the treatment of diabetes it is well to establish exactly what is meant by "dietary fibre". It has been defined as the plant polysaccharides and lignin which are resistant to hydrolysis by the digestive system [8]. There are four major constituents to "fibre": cellulose, hemicellulose, pectin and lignin. The first three are also termed "unavailable carbohydrates" i. e. they are unavailable in a nutritional sense. Cellulose is present in all plants, the amount varying with species and age of the plant. Although man is unable to hydrolyse cellulose, approximately $15 \%$ can be broken down by gut bacteria. Hemicullulose is widely distributed, particularly in young plants. It consists of a large variety of polysaccharides containing sugar residues such as xylose, arabinose, glucose and mannose. Approximately $85 \%$ of hemicellulose is degraded in the colon, although the products are obviously not available for absorption. Pectins are also predominently found in young plants, the main input in man coming from citrus fruits. They are hydrophilic and adhesive. There are many other minor components of "dietary fibre". These include plant gums, gels and mucillages. Strictly these are "unabsorbable carbohydrates" but not fibres at all. They include the galactomannans which are common storage polysaccharides in legumes. These are used widely in industry [9]: 10 million kilograms/year in the paper industry, as stabilisers in ice cream, and even has a component of certain explosives (which is not so incongruous for those of us who have consumed them).

Most nutritional studies "fibre" have ignored the individual components, but have measured the residue found after treatment of food with dilute acid and alkali: this is referred to as "crude fibre" and does not include either pectins or $80 \%$ of the hemicellulose which are degraded in the process. Recent studies have been more precise [10] and hopefully information will now be forthcoming on the relative importance of the specific components of "fibre".

What relationship does fibre consumption bear to diabetes? Trowell has suggested that dietary-fibre depleted starchy foods are conducive the the development of diabetes mellitus in susceptible genotypes [7]. Much of his argument was based on the Western diet input of some $4 \mathrm{~g}$ of fibre per day compared with the African intake of $10 \mathrm{~g}-25 \mathrm{~g}$, and the very differing frequency of diabetes in these two situations. Much further work will be required, however, to substantiate Trowell's hypothesis. It is possible that obesity with its known association with mild 
diabetes is related to a low intake of dietary fibre. A high fibre input dilutes calories and more masticatory effort is required to obtain the same caloric input [11]. More work has been done, however, on the effects of fibre in patients with already established diabetes.

It has long been recognized that the rise in blood glucose and serum insulin concentrations is less when sugars are consumed with complex polysaccharides than when the pure sugar alone is taken $[12,13]$. More recently the specific effects of increasing dietary fibre content have been examined. Douglas [14] has described the case of two insulin-dependet diabetics in whom insulin therapy was decreased or stopped altogether, when they were given diets comprising mainly raw and uncooked food. Kiehm et al. [15] obtained similar dramatic results. They fed high fibre, high carbohydrate diets to thirteen diabetic men, five of whom were taking 15-28 units insulin/ day, three were taking $40-55$ units insulin/day while the remaining five were being treated with sulphonylureas. After two weeks on the high fibre diet the sulphonylureas could be stopped in all five patients, and insulin was discontinued in four of the five patients in the low insulingroup. No effect, however, was noted in the three patients taking the larger dose of insulin. In a five day study by another group [16] in which $25 \mathrm{~g}$ guar was added to the diet of seven insulin-dependent patients there was a significant fall in urine glucose excretion although no change in insulin therapy was reported.

Recent studies have concentrated more on the effects of specific components of fibre. Jenkins et al. [17] gave $12 \mathrm{~g}$ of fibre or fibre analogues as either guar, gum tragacanth, pectin, methylcellulose, wheat bran or cholestyramine together with $50 \mathrm{~g}$ glucose and $25 \mathrm{~g}$ xylose to healthy individuals. In all cases the glucose and insulin responses were flattened although the most significant results were obtained with guar. There was a highly significant correlation between "fibre" viscosity and the degree of suppression of glucose and insulin. At the same time xylose absorption was delayed although after 6 to 8 hours the total amount excreted was the same. There was thus delay in absorption but importantly no malabsorption. Similar findings have been noted when guar was added to meals given to noninsulin dependent diabetics [18] and when $10 \mathrm{~g}$ guar was added to breakfasts taken by six insulin-dependent diabetics [19]. In the latter case as well as in normal subjects there was not only diminished postprandial glycaemia but also a marked fall in GIP release [20]. The mechanism of the diminished insulin response in normal subjects and mild diabetics may not just therefore be simply due to a lower B-cell glucose stimulus but may involve gut hormones as well. This view is supported by the findings of Blotman et al. [21] who showed a more profound lowering of insulin levels than of glycaemia when adding bran to a standard glucose test in mild diabetics.

There are two possible important conclusions from these studies. The first is that total insulin therapy may be decreased by adding specific fibre components to the diets of diabetics and indeed in selected patients it may be possible to stop insulin therapy altogether. Perhaps more important, however, is the possibility of improving diurnal blood glucose profiles. Insulin given subcutaneously is absorbed slowly compared with the normal pancreatic insulin response to a meal. There thus tends to be a hyperglycaemic phase in the first one or two hours following a meal in an insulin-dependent diabetic, followed by a hypoglycaemic rebound as insulin absorption reaches in maximum. This is illustrated by the importance of mid-morning and mid-afternoon snacks in diabetics, and the relative frequency of late morning hypoglycaemia in patients taking "fast"-acting insulins. Guar slows glucose absorption and therefore the glucose profile should more closely match the pattern of insulin absorption. Less insulin should be necessary and fewer peaks and troughs in the glucose profile should be encountered. This hypothesis remains to be tested, but is potentially of critical importance if we accept, as we surely must, that attainment of normoglycaemia is a major aim in the therapy of diabetes.

The other potentially important aspect of high fibre diets in diabetics is their relation to ischaemic heart disease (IHD). Several epidemiological studies have been reported relating the incidence of IHD to dietary fibre input. Thus Morris et al. [22] in a retrospective study of 357 healthy working men, aged 30-67 years, showed a clear association of low total fibre intake with IHD, and a marked freedom from IHD in those taking high fibre diets. The main association was with fibre derived from cereals rather than that contained in legumes, pulses and nuts. Other studies have concentrated on the effects of varying dietary fibre intake on serum cholesterol and triglycerides. Bran is strikingly without effect on serum cholesterol and triglyceride levels in subjects with normal pre-test values, but does have a hypotriglyceridaemic action if values are high before treatment [23]. In contrast the "gelling" fibres, pectin and guar, have been shown to decrease plasma cholesterol levels when given in large amounts chronically [22]. In diabetics Kiehm and his colleages [8] showed a fall in serum cholesterol and triglyceride levels when they were given high carbohydrate high fibre diets, but it should be noted that the control diet contained considerably more total fat than the test diet $(83 \mathrm{~g}$ vs $23 \mathrm{~g}$ ).

Obviously the type of fibre is important. In normal subjects Raymond et al. [25] found no effect on cholesterol using cholesterol free diets enriched with a mixture of fibrous fibres. When cholesterol was 
added to the diet, a small hypocholesterolaemic effect of fibre was noted in the LDL fraction. In contrast Durrington et al. [26] found a clear effect of pectin on LDL cholesterol. More studies are now required where specific fibres are looked at in relation to specific lipoprotein fractions. If dietary fibre is to be important in treating hyperlipidaemia, and indeed in preventing ischaemic heart disease (and there is a quantum jump between these two effects) then it is vital, that we know precisely which cholesterol and triglyceride fractions are affected. There is also a clear need for a good carefully controlled prospective study of the effects of dietary fibre on macroangiopathy and on lipids in a diabetic population.

One remaining problem concerns palatability. Many studies, particularly the retrospective epidemiological ones, have concerned themselves purely with total fibre input. Recent work, however, suggests that the "gelling" fibre are more efficacious, certainly in terms of glycaemia effects and probably with respect to lowering of blood lipids as well. Substances such as guar are certainly of great use as binding agents in industry but are unpleasant to consume in bulk. Even when carefully prepared in soups or bread they can leave an unpleasant after-taste and can cause profound and socially inconvenient flatus. Further work is urgently required to produce palatable alternatives. This should be possible now that we have more information on the physicochemical characteristics required for effective action.

In conclusion, certain components of dietary fibre may be of use in the treatment of diabetic patients. Blood glucose profiles may be improved and elevated lipid levels lowered. Note should be made, however, of potential hazards such as malabsorption of calcium, iron and zinc $[27,28]$ and more basic information is required on the overall nutritional effects of different dietary fibres. Certainly, ignoring journalistic rhetoric and concentrating solely on experimental results, there is enough suggestive data to warrant proper prospective assessment of dietary fibre in not only the treatment of diabetes mellitus, but also in prophylaxis against IHD.

\section{References}

1. Leader: Dietary fibre. Lancet 1977 II, 337-338

2. Leader: Dietary fibre and plasma lipids. Lancet 1975 II, 353-355

3. Leader: Food and fibre. Br. Med. J. 1977 II, 418

4. Eastwood, M. A.: Dietary fibre and serum lipids. Lancet 1969 II, 1222-1225

5. Burkitt, D. P.: Epidemiology of cancer of the colon and rectum. Cancer 28, 3-13 (1971)

6. Burkitt, D. P., Walter, A. R. P., Painter, N. S.: Diet, fibre and disease. J. A. M. A. 229, 926-932 (1972)

7. Trowell, H.C.: Dietary-fiber hypothesis of the etiology of diabetes mellitus. Diabetes 24, 762-764 (1975)

8. Trowell, H.C.: Dietary fibre, ischaemic heart disease and diabetes mellitus. Proc. Nutr. Soc. 32, 151-157 (1973)
9. Goldstein, A. M., Alter, E. N., Seaman, J. K., In: R. L. Whistler, J. N. Bertram (Ed.): Industrial Gums, pp. 303-322. New York and London: Academic Press 1973

10. Southgate, D. A. T., Bailey, B., Collinson, E., Walker, A. F. A guide to calculating intakes of dietary fibre. J. Hum. Nutr. 30, 303-313 (1976)

11. Heaton, K. W.: Food fibre as an obstacle to energy intake. Lancet 1973 II, 1418-1421

12. Swan, D. C., Davidson, P., Albrink, M. J.: Effect of simple and complex carbohydrates on plasma non-esterified fatty acids, plasma sugar, and plasma insulin during oral carbohydrate tolerance tests. Lancet 1966 I, 60-63

13. Conn, J.W., Newburgh, L. H.: Glycemic response to isoglucogenic quantities of protein and carbohydrate. J. Clin. Invest 15, 665-671 (1936)

14. Douglas, J. M.: Raw diet and insulin requirement. Ann. Intern. Med. 82, 61-63 (1975)

15. Kiehm, T. G., Anderson, J. W., Ward, K.: Beneficial effect of a high carbohydrate, high fiber diet on hyperglycemic diabetic man. Am. J. Clin. Nutr. 29, 895-899 (1976)

16. Jenkins, D. J. A., Hockaday, T. D. R., Haworth, R., Apling, E. L., Wolever, T. M. S., Leeds, A. R., Bacon, S., Dilawari, J.: Treatment of diabetes with guar gum. Reduction in urinary glucose loss in diabetics. Lancet 1977 II, 779-780

17. Jenkins, D. J. A., Wolever, T. M. S., Leeds, A. R., Gassull, M. A., Haisman, P., Dilawari, J., Goff, D. V., Metz, G. L., Alberti, K. G. M. M.: Dietary fibre, fibre analogues and glucose tolerance: importance of viscosity. Br. Med. J. 1978 II, 1392-1394

18. Jenkins, D. J. A., Leeds, A. R., Gassull, M. A., Wolever, T.M.S., Goff, D.V., Alberti, K. G. M.M., Hockaday, T. D. R.: Unabsorbable carbohydrates in diabetes: decreased postprandial hyperglycaemia. Lancet 1976 II, 172-174

19. Goulder, T. J., Alberti, K. G. M. M., Jenkins, D. J. A.: Effect of added fiber on the glucose and metabolic response to a mixed meal in normal and diabetic subjects. (Submitted for publication)

20. Morgan, L.M., Goulder, T.J., Tsiolakis, D., Marks, V., Alberti, K. G. M. M.: The effect of unabsorbable carbohydrate on gut hormones: modification of post-prandial GIP secretion by guar. (Submitted for publication)

21. Blotman, M. J., Cartry, E., Monnier, L., Mirouze, J.: Les fibres alimentaires. Sem. Hop. Ther. 53, 285- 293 (1977)

22. Morris, J. N., Mart, J.W., Clayton, D. G.: Diet and heart: a postcript. Br. Med. J. 1977 II, 1307-1314

23. Truswell, A. S., Kay, R. M.: Bran and blood lipids. Lancet 1976 I, 367

24. Jenkins, D. J. A., Leeds, A. R., Newton, C., Cummings, J. H.: Effect of pectin, guar gum and wheat fibre on serum cholesterol. Lancet 1975 I, 116-117

25. Raymond, T. L., Connor, W. E., Lin. D. S., Warner, S., Fry, M. M., Connor, S. L.: The interaction of dietary fibres and cholesterol upon plasma lipids and lipoproteins, stool balance, and bowel function in human subjects. J. Clin. Invest. 60 1429-1437 (1977)

26. Durrington, P. N., Manning, A. P., Bolton, C. H., Hartog, M.: Effect of pectin on serum lipids and lipoproteins, whole-gut transit-time, and stool weight. Lancet 1976 II, 394-396

27. Rheinhold, J. G., Faradji, B., Abadi, P., Ismail Beizi, F.: Decreased absorption of calcium, magnesium and phosphorus by humans due to increased fibre and phosphorus consumed as white bread. J. Nutr. 106, 493 (1976)

28. Widdowson, E. M., McCance, R. A.: Iron exchanges of adults on white and brown bread diets. Lancet 1942 I, 588-591

Dr. T. J. Goulder

Faculty of Medicine

Chemical Pathology and Human Metabolism

Level D

South Laboratory and Pathology Block

General Hospital

Southampton, S09 4XY

England 